\title{
The effects of proliferating cell nuclear antigen and $p 53$ in patients with oral squamous cell carcinoma: a systematic review and meta-analysis
}

\author{
Rui Liu", Kunjun Sun", Yuanda Wang, Yunxian Jiang, Jianyong Kang, Hong Ma \\ Department of Oral and Maxillofacial Surgery, Affiliated Hospital of Guizhou Medical University, Guiyang, China \\ Contributions: (I) Conception and design: R Liu, K Sun, H Ma; (II) Administrative support: Y Wang, Y Jiang, J Kang; (III) Provision of study materials \\ or patients: R Liu, K Sun; (IV) Collection and assembly of data: R Liu, K Sun, Y Wang, Y Jiang, J Kang; (V) Data analysis and interpretation: R Liu, \\ K Sun; (VI) Manuscript writing: All authors; (VII) Final approval of manuscript: All authors. \\ \#These authors contributed equally to this work. \\ Correspondence to: Hong Ma. Department of Oral and Maxillofacial Surgery, Affiliated Hospital of Guizhou Medical University, No. 28 Guiyi Street, \\ Guiyang, China. Email: mahong1966@126.com.
}

\begin{abstract}
Background: To evaluate the effect of proliferating cell nuclear antigen $(P C N A)$ and $p 53$ in patients with oral squamous cell carcinoma (OSCC).

Methods: Multiple databases, including PubMed, Embase, Cochrane library, and China National Knowledge Database, were searched for relevant studies and full-text articles that evaluated the effect of PCNA and $p 53$ in patients with OSCC. Review Manager 5.2 was adopted to estimate the impact of the results among the selected articles. Forest plots, NOS table, sensitivity analysis, and bias analysis were also conducted.

Results: In total, nine eligible studies satisfied the included criteria. High PCNA expression ( $>50 \%)$ was significantly more prevalent in OSCC than low PCNA expression (<50\%) (OR =3.88; 95\% CI: 2.04-7.37; $\left.\mathrm{P}<0.0001 ; \mathrm{I}^{2}=0 \%\right)$. However, there was no significant difference between $p 53$ and OSCC $(\mathrm{OR}=1.60 ; 95 \% \mathrm{CI}$ : 0.18-14.63; $\left.\mathrm{P}=0.68 ; \mathrm{I}^{2}=86 \%\right)$. Low PCNA expression had a higher 5 -year overall survival in OSCC patients than high PCNA expression (OR =0.47; 95\% CI: 0.27-0.80; $\left.\mathrm{P}=0.005 ; \mathrm{I}^{2}=41 \%\right)$. Meanwhile, $p 53$ negative had a higher 5 -year overall survival than $p 53$ positive $\left(\mathrm{OR}=0.20 ; 95 \% \mathrm{CI}: 0.10-0.42 ; \mathrm{P}<0.0001 ; \mathrm{I}^{2}=0 \%\right)$. There was no difference between high and low PCNA in terms of metastasis (OR $=0.80$ with 95\% CI: 0.18-3.45, $\mathrm{I}^{2}=63 \%$, $\mathrm{P}$ of over effect $=0.76$ ). The overall results showed no difference between $p 53$ and metastasis (OR $=0.38$ with $95 \%$ CI: $0.13-1.10, \mathrm{I}^{2}=0 \%$, P of over effect $\left.=0.07\right)$.
\end{abstract}

Discussion: $P C N A$ and $p 53$ might be suitable for prognostic and survival evaluation in OSCC patients.

Keywords: Oral squamous cell carcinoma (OSCC); proliferating cell nuclear antigen (PCNA); p53; meta-analysis

Submitted Oct 29, 2021. Accepted for publication Dec 02, 2021.

doi: $10.21037 /$ atm-21-6133

View this article at: https://dx.doi.org/10.21037/atm-21-6133

\section{Introduction}

Globally, head and neck cancers are estimated to comprise 500,000 patients with squamous cell carcinoma (SCC) every year. SCC is the most common malignant tumor of the head and neck, accounting for $90 \%$ (1). Due to the location of oral SCC (OSCC) in the body, the social and medical impact of these lesions is more significant than other more common tumors. OSCCs are close to vital structures in the head and neck, making treatment difficult, and the results are often severely deformed (2). Part of the reason for the poor prognosis (5-year survival of approximately $50 \%$ ) and high recurrence rate (about 645,000 per year) of OSCC is the lack of an accurate and clinically applicable staging system. Also, the current clinical diagnosis system 
for predicting the local control and survival rate of OSCC is limited (3).

As a marker of cell proliferation, $P C N A$ is considered a convenient tool for quickly assessing the proportion of proliferating cells in tumors. PCNA is a nuclear non-histone antigen that appears in the nucleus in the late G1 phase. It increases in the $\mathrm{S}$ phase and declines in the G2 and $M$ phases. $P C N A$ is a $36 \mathrm{kda}$ molecule that plays an essential role in nucleic acid metabolism due to the replication and repair mechanism (4). It serves as an accessory protein for DNA polymerase; it is needed to synthesize S-phase chromosomal DNA and interact with cellular proteins involved in regulating the cell cycle and checkpoint control (5). Some studies have suggested that PCNA expression is a marker of abnormal cell proliferation and could be used as a reference index for early cancer diagnosis $(4,5)$.

The tumor suppressor gene, $p 53$, is a genetic biomarker that regulates cell growth and proliferation. The wildtype $\mathrm{p} 53$ protein controls the cell cycle's progression by acting as transcription factors for multiple genes, which induces transcriptional regulation of the cyclin-dependent kinase inhibitor p21 (6). The stability and overexpression of the $p 53$ gene might be related to $p 53$ gene mutation or genotoxic stress, and $p 53$ gene changes are the most common genetic abnormality in many cancers. In OSCC, multiple studies have shown that overexpression of $p 53$ plays a vital role in the development of OSCC (7). $p 53$ is an important anticancer gene; its wild type can induce apoptosis of cancer cells and prevent canceration, and could also help cells repair gene defects (3). In addition, it was reported that there was significant correlation between the expression level of $\mathrm{p} 53$ protein and postoperative survival time of oral squamous cell carcinoma and the expression of PCNA protein was closely related to the risk of OSCC, and could be used as an important index to judge the prognosis of OSCC patients (5-7).

In recent years, the value of $P C N A$ and $p 53$ in OSCC has been noted (7), but the detailed role of PCNA and $p 53$ in OSCC has not been fully elucidated. Herein, we conducted a meta-analysis to evaluate the effects of PCNA and $p 53$ in patients with OSCC. This research is a comprehensive analysis from four aspects and can be a supplement for this topic. In this research, we analyzed the association between oral squamous cell carcinoma and $p 53$ or PCNA, respectively. We present the following article in accordance with the PRISMA reporting checklist (available at https:// dx.doi.org/10.21037/atm-21-6133).

\section{Methods}

\section{Literature search strategy}

We searched articles published between January 2000 and March 2020 for PCNA and $p 53$ in OSCC patients in the PubMed, Embase, Cochrane database, and China National Knowledge databases using the following strategy: (oral OR mouth OR tongue) AND (cancer* OR squamous cell carcinoma* OR neoplasm* OR tumor*) AND (PCNA OR p53). There were no restrictions on the publication language in the literature search. To maximize the specificity and sensitivity of our search, we checked the research reference list to seek other relevant research that were not found through the search strategy.

\section{Study selection}

\section{Inclusion criteria and exclusion criteria}

We used the following inclusion criteria for our research: (I) studies with case-control design; (II) studies evaluating the effect of PCNA and $p 53$ in prognosis, survival, and metastasis; (III) articles containing eligible data; and (IV) articles with available full text. Research meeting any one of the following conditions was excluded: (I) studies with overlapping data or overlapping review articles; (II) studies involving patients with other head and neck tumors, and (III) articles involving other biomarkers for OSCC patients.

\section{Data extraction and quality assessment}

Two commentators independently scanned the full texts of the manuscripts. They extracted the following data from each eligible study: first author's name, patient's age and gender, country of origin, year of publication, sample size, and the study period of each article. The Cochrane risk of the bias assessment tool, which is a comprehensive tool to consider multiple biases, was used to evaluate the methodological quality of the studies.

\section{Statistical analysis}

We used Review Manager (version 5.2, Cochrane Collaboration, 2011) to assess the impact of the results in selected reports. For continuous outcomes, the mean difference was calculated by the average difference. Heterogeneity was evaluated by the $\mathrm{I}^{2}$ statistic, which is the 
Identification of studies via databases and registers

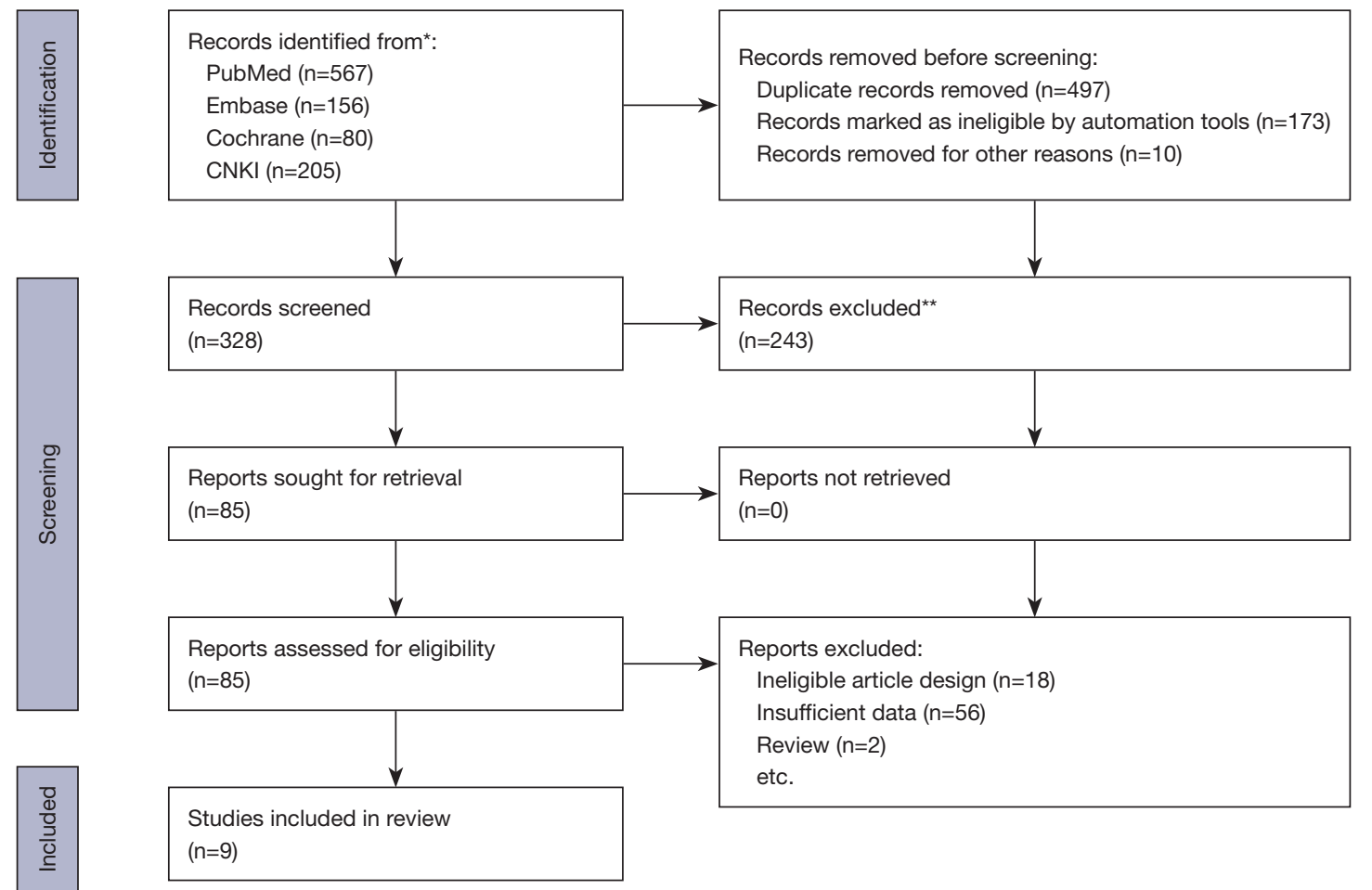

Figure 1 Flow diagram of the study selection. This flow diagram shows the process of study inclusion and exclusion in this meta-analysis. *, consider, if feasible to do so, reporting the number of records identified from each database or register searched (rather than the total number across all databases/registers); **, if automation tools were used, indicate how many records were excluded by a human and how many were excluded by automation tools.

percentage of heterogeneity among studies in the absolute difference and a quantitative measure of inconsistency in research. We confirmed that studies with an $\mathrm{I}^{2}$ of $25-50 \%$ were considered to have low heterogeneity, studies with an $\mathrm{I}^{2}$ of $50-75 \%$ were deemed to be medium heterogeneity, and studies with $\mathrm{I}^{2}>75 \%$ were considered to have high heterogeneity. If $\mathrm{I}^{2}>50 \%$, the potential sources of heterogeneity were examined by sensitivity analysis, which omits one study in each round and investigates the impact of a single portfolio survey estimation. Also, when heterogeneity was observed, the random effects model was used; otherwise, the fixed effects model was used. We used funnel charts, Begger's test, and Egger's test to check for potential publication bias.

\section{Results}

\section{Search process}

The electronic search retrieved 328 articles. After careful reading, 85 papers have met the preliminary standard. Upon further screening, 76 articles were excluded because of duplication, irrelevant studies, incomplete data, and incomplete comparison. Finally, nine papers were selected for analysis. Figure 1 displays a flowchart of the search process, highlighting the identification, inclusion, and 
Table 1 Characteristics of studies included in this meta-analysis

\begin{tabular}{|c|c|c|c|c|c|c|c|}
\hline Study & Year & Language & Country & Age (years) & Groups & $\mathrm{n}$ & Years of onset \\
\hline Fernanda (8) & & & & & Low PCNA & 17 & \\
\hline \multirow[t]{2}{*}{ Kato (9) } & 2011 & English & Japan & $66.8 \pm 10.1$ & High PCNA & 11 & 2002 to 2006 \\
\hline & & & & & Low PCNA & 48 & \\
\hline Keum (10) & & & & & Low PCNA & 15 & \\
\hline \multirow[t]{2}{*}{ Lee (11) } & 2005 & English & China & $47 \pm 18.5$ & High PCNA & 38 & 1995 TO 2001 \\
\hline & & & & & Low PCNA & 38 & \\
\hline Mallick (12) & 2010 & English & India & $55 \pm 10.2$ & High PCNA & 20 & 1998 to 2003 \\
\hline Monteiro (13) & & & & & Low PCNA & 34 & \\
\hline \multirow[t]{2}{*}{ Myoung (14) } & 2006 & English & Korea & $58.2 \pm 10.2$ & High PCNA & 59 & 1996 to 2001 \\
\hline & & & & & Low PCNA & 54 & \\
\hline \multirow[t]{2}{*}{ Stenner (15) } & 2012 & English & Germany & $59.4 \pm 1.3$ & High PCNA & 12 & 1986 to 2006 \\
\hline & & & & & Low PCNA & 12 & \\
\hline \multirow[t]{2}{*}{ Watanabe (16) } & 2010 & English & Brazil & $60.5 \pm 8.3$ & High PCNA & 19 & 1996 to 2002 \\
\hline & & & & & Low PCNA & 20 & \\
\hline
\end{tabular}

PCNA, proliferating cell nuclear antigen.

exclusion (including reasons) process.

\section{Characteristics of included studies}

Detailed characteristics of the included studies are presented in Table 1. All of the included studies were published between 2005 and 2020. The sample size ranged from 20 to 113 . In total, 223 patients were in the high $P C N A$ group and 257 patients were in the low $P C N A$ group.

\section{Quality assessment}

Since the included articles were case-control studies, we used the Newcastle-Ottawa Scale (NOS) table to evaluate the risk of patient selection problems in nine trials (Table 2). Four of the nine included articles had 9 stars, and the other five had 8 stars, which demonstrated that included papers were good quality ( $>6$ stars was considered to indicate good research quality).

\section{Heterogeneity analysis}

Heterogeneity analysis of the prognostic value of PCNA and $p 53$ in OSCC

Since five of the nine included studies did not report on PCNA level, comprehensive analysis was performed on the other four articles. As shown in Figure $2 \mathrm{~A}$, $\mathrm{I}^{2}=0 \%$, and thus a fixed effects model was adopted. The results showed high PCNA expression (event/total: 61/87) was significantly more prevalent than low PCNA expression (33/86) in OSCC [odds ratio (OR) $=3.88 ; 95 \%$ confidential interval (CI): 2.04-7.37; $\mathrm{P}<0.0001 ; \mathrm{I}^{2}=0 \%$, Figure 2A]. However, only two studies reported on $p 53$ expression in OSCC. As shown in Figure $2 B, \mathrm{I}^{2}=86 \%$, and therefore a random effects model was used. The result suggested that there was no significant difference between $p 53$ positive $(33 / 55)$ and $p 53$ negative $(22 / 55)$ in OSCC $\left(\mathrm{OR}=1.60 ; 95 \%\right.$ CI: $0.18-14.63 ; \mathrm{P}=0.68 ; \mathrm{I}^{2}=86 \%$, Figure 2B). 
Table 2 Newcastle-Ottawa Scale table of included studies

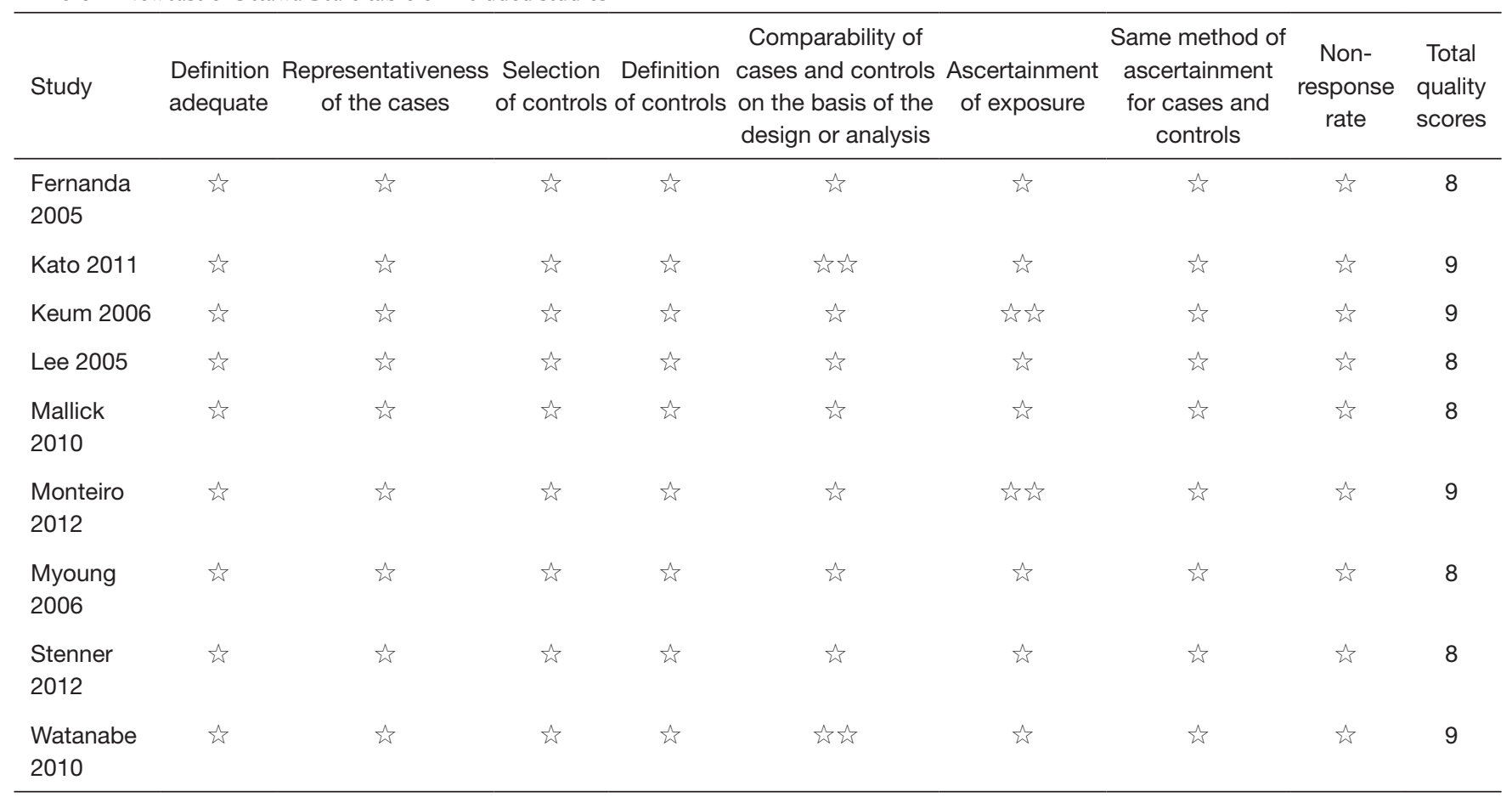

is, medium quality ; is , high quality. The higher the quality score is, the better quality of article is.

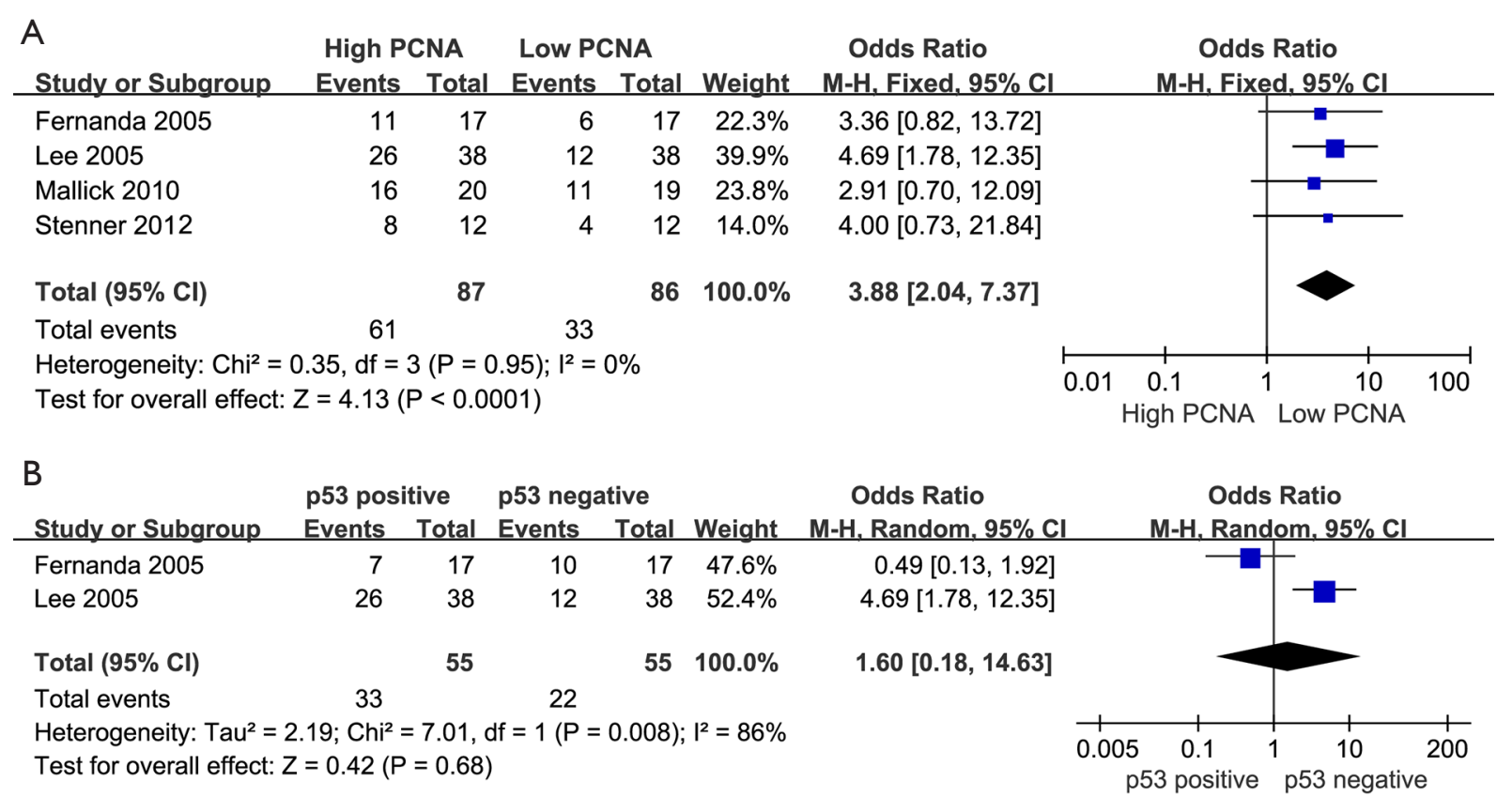

Figure 2 Forest plots of the prognostic value of PCNA and $p 53$ in OSCC. (A) OSCC patients' PCNA was compared; (B) OSCC patients' $p 53$ was contrasted. PCNA, proliferating cell nuclear antigen; $p 53, p 53$ gene; OSCC, oral squamous cell carcinoma. 
A

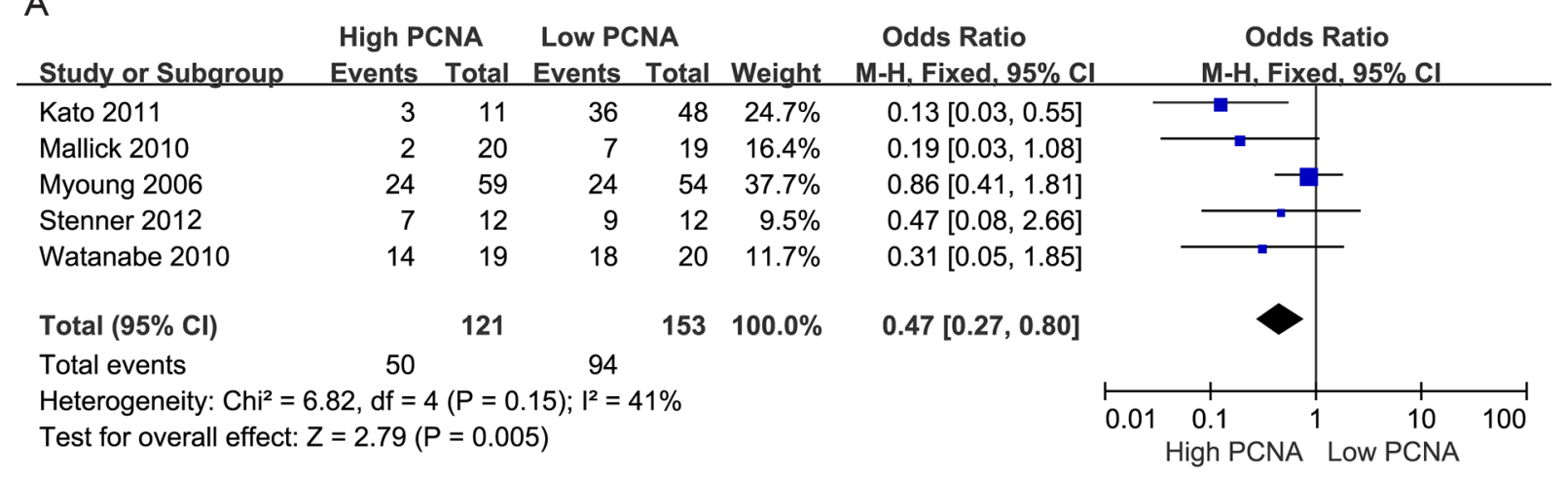

B

\begin{tabular}{|c|c|c|c|c|c|}
\hline & p53 posi & tive & p53 nege & tive & \\
\hline Study or Subgroup & Events & Total & Events & Total & Weic \\
\hline Kato 2011 & 3 & 11 & 36 & 48 & 30.8 \\
\hline Monteiro 2012 & 12 & 42 & 23 & 34 & \\
\hline Stenner 2012 & 7 & 12 & 9 & 12 & \\
\hline Total $(95 \% \mathrm{Cl})$ & & 65 & & 94 & 100 \\
\hline Total events & 22 & & 68 & & \\
\hline Heterogeneity: Chi & 31, $d f=2$ & $(P=0$. & 52); $1^{2}=0^{c}$ & & \\
\hline
\end{tabular}

Odds Ratio Odds Ratio

M-H, Fixed, $95 \% \mathrm{Cl} \quad \mathrm{M}-\mathrm{H}$. Fixed, $95 \% \mathrm{Cl}$

$0.13[0.03,0.55]$

$0.19[0.07,0.51]$

$0.47[0.08,2.66]$

$0.20[0.10,0.42]$

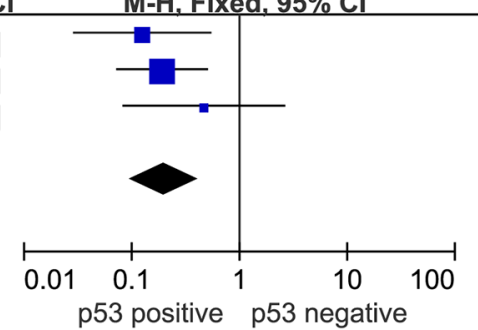

Figure 3 Forest plots of the value of PCNA and $p 53$ on the 5-year overall survival among patients with OSCC. (A) OSCC patients' 5-year overall survival with different PCNA levels was compared; (B) OSCC patients' 5 -year overall survival with different $p 53$ was contrasted. $P C N A$, proliferating cell nuclear antigen; $p 53, p 53$ gene; OSCC, oral squamous cell carcinoma.

Heterogeneity analysis regarding the value of $P C N A$ and $p 53$ on the 5 -year overall survival among patients with OSCC

As shown in Figure 3A, five of the nine studies reported on PCNA and the 5-year overall survival of OSCC patients. Since the $\mathrm{I}^{2}$ value was low, the fixed effects model was used. The results showed that low $P C N A$ expression had a higher 5 -year overall survival in OSCC patients (94/153) than high PCNA expression (50/121) (OR $=0.47$; 95\% CI: 0.27-0.80; $\mathrm{P}=0.005 ; \mathrm{I}^{2}=41 \%$, Figure $3 A$ ). As for $p 53$, a fixed effects model was used for heterogeneity analysis, which showed that $p 53$ negative $(68 / 94)$ had a higher 5 -year overall survival than $p 53$ positive (22/65) (OR $=0.20$; $95 \%$ CI: 0.10 $0.42 ; \mathrm{P}<0.0001 ; \mathrm{I}^{2}=0 \%$, Figure $3 B$ ).

Heterogeneity analysis on the role of PCNA and $p 53$ in metastasis

We used three articles (3/9) for PCNA and two articles (2/9) for $p 53$ to conduct heterogeneity analysis. The heterogeneity test results showed that we needed a random effects model to analyze the data ( $\mathrm{OR}=0.80$ with $95 \% \mathrm{CI}$ :
$0.18-3.45, \mathrm{P}$ of heterogeneity $=0.07, \mathrm{I}^{2}=63 \%, \mathrm{Z}=0.30, \mathrm{P}$ of over effect $=0.76$, Figure $4 A$ ). There was no difference in the overall effect of high (18/38) and low (28/51) PCNA on metastasis (Figure 4A). A fixed effects model was used to evaluate $p 53$, and also showed no difference between $p 53$ and metastasis $(\mathrm{OR}=0.38$ with $95 \% \mathrm{CI}: 0.13-1.10, \mathrm{P}$ of heterogeneity $=0.71, \mathrm{I}^{2}=0 \%, \mathrm{Z}=1.79, \mathrm{P}$ of over effect $=0.07$, Figure $4 B)$.

\section{Sensitivity analysis and publication bias}

According to heterogeneity analysis, the heterogeneity of $P C N A$ in OSCC was low $\left(\mathrm{I}^{2}=0 \%, \mathrm{P}<0.0001\right)$. This might be attributed to the different results of each study. When Lee et al. (11) from 2005 was excluded, the $\mathrm{I}^{2}$ did not change, while the $\mathrm{P}$ value of heterogeneity changed from 0.95 to 0.96 (Figure 5). The sensitivity analysis indicated that the results in this article were robust.

We performed a funnel plot for PCNA in OSCC. Four studies were included in the plot. The standard error of or logarithm is ordinate, and the image symmetry is the 


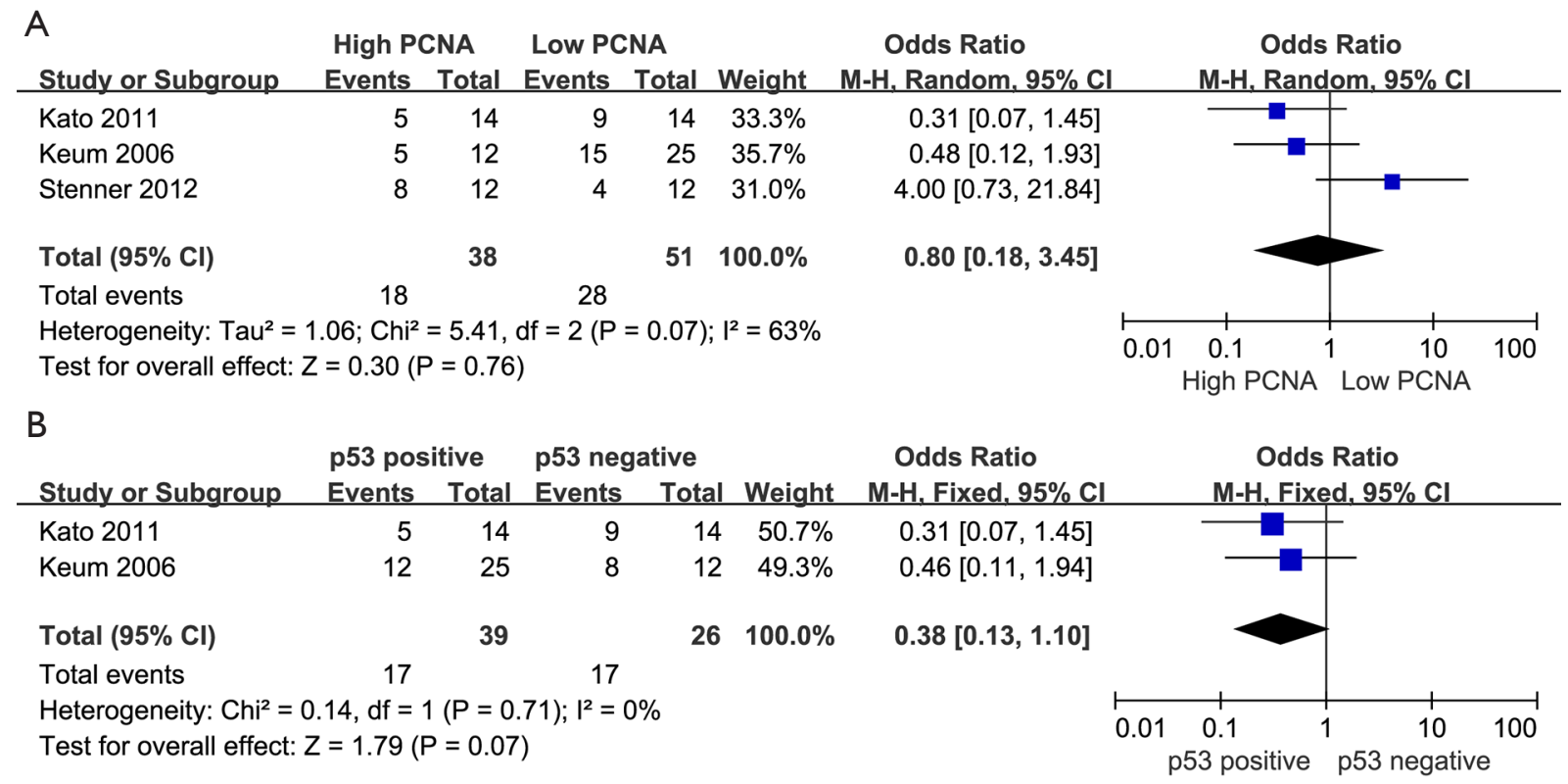

Figure 4 Forest plots of the role of $P C N A$ and $p 53$ on metastasis. (A) OSCC patients' metastasis with different $P C N A$ levels was compared; (B) OSCC patients' metastasis with different $p 53$ was contrasted. PCNA, proliferating cell nuclear antigen; $p 53$, $p 53$ gene; OSCC, oral squamous cell carcinoma.

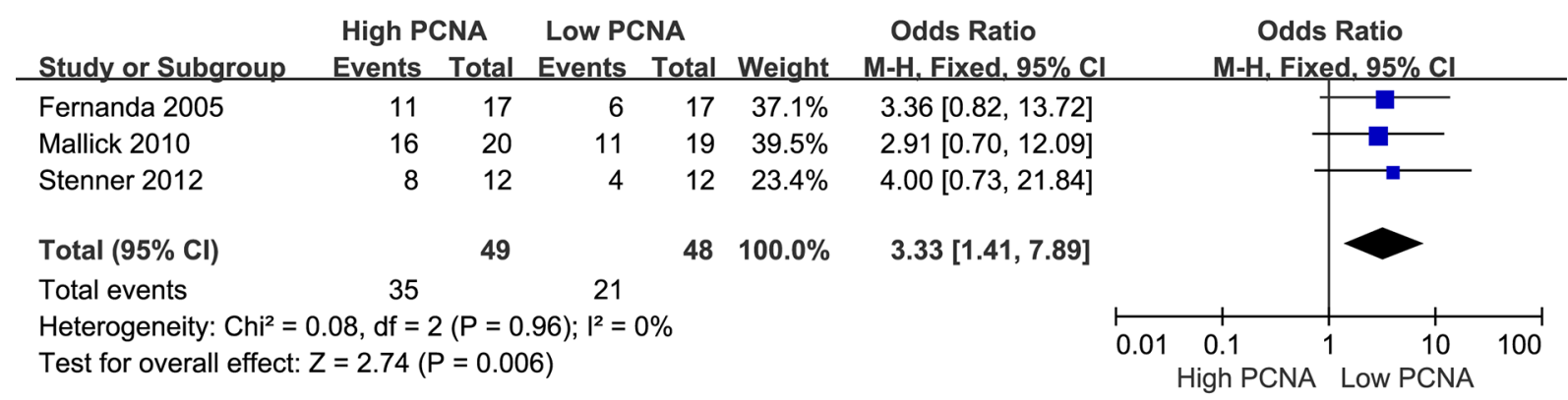

Figure 5 Sensitivity analysis forest plots of the prognostic value of PCNA in OSCC. PCNA, proliferating cell nuclear antigen; OSCC, oral squamous cell carcinoma.

basis of judging publication bias. When it is symmetrical, publication bias is slight; when it is asymmetrical, publication bias is significant (7). To some extent, the result indicated that there existed slight publication bias, since the symmetrical characteristic of the funnel plot was good (Figure 6). The result of Begger's test suggested that no significant evidence of potential publication bias existed $(\mathrm{z}=1.15, \mathrm{P}=0.101)$, and Egger's test also indicated that no significant evidence of possible publication bias existed $(\mathrm{t}=1.27, \mathrm{P}=0.215)$.

\section{Discussion}

Our results showed that high $P C N A$ expression was significantly more prevalent in OSCC than low PCNA expression, which indicated that $P C N A$ might have predictive value for OSCC. Sajeevan (17) stated that the functional change of $P C N A$ activity is a joint genetic event in various cancers and an effective marker of cell proliferation. It could be used to determine the histological grade, recurrence rate, and prognosis of head and neck 


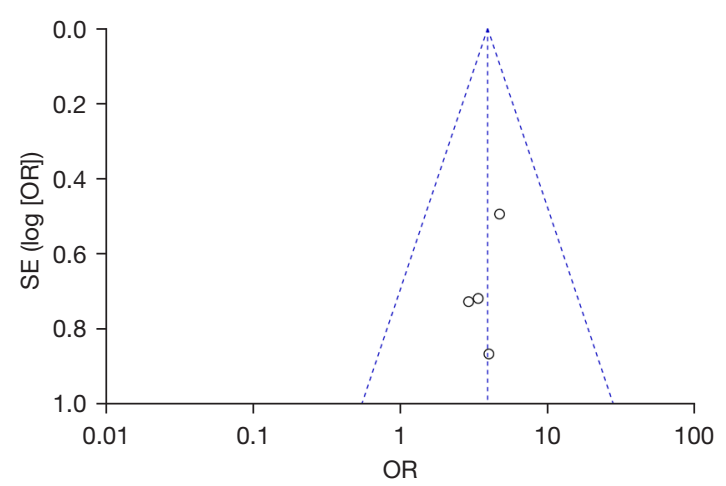

Figure 6 Funnel plot of the prognostic value of PCNA and $p 53$ in OSCC. $P C N A$, proliferating cell nuclear antigen; $p 53, p 53$ gene; OSCC, oral squamous cell carcinoma.

cancers. Overexpression of PCNA is also associated with chemotherapy or radiation therapy (17). However, the relationship between PCNA changes and cervical lymph node metastasis of oral tongue cancer remains unclear. In the analysis of the 5-year overall survival and PCNA, low $P C N A$ expression had a higher 5 -year overall survival in OSCC patients than high PCNA expression. These findings demonstrated that low $P C N A$ might be an influencing factor for OSCC patients' 5 -year overall survival. Furthermore, the results regarding the role of $P C N A$ in the metastasis of OSCC patients suggested that PCNA is not valuable for determining metastasis.

The analysis also showed that $p 53$ could be a potential indicator for the 5-year overall survival of OSCC patients, but does not appear to have predictive value for OSCC. Zhong et al. (5) reported that $p 53$ gene mutations and overexpression of mutant $\mathrm{p} 53$ proteins play an essential role in the occurrence and loss of apoptosis in various human cancers. Simultaneously, $p 53$ gene mutations have been increasingly found in several poorly differentiated head and neck cancers, including oral cancer (18). There are also changes in $p 53$ that are associated with aggressive laryngeal and pharyngeal phenotype tumor recurrence (19). Overexpression of $p 53$ is associated with a higher risk of oral lymph node metastasis, and is a marker of poor prognosis for oral squamous cell cancer (20). However, these correlations have not been further confirmed by other studies. The results about the role of $p 53$ in metastasis among OSCC patients showed that $p 53$ might not have any value for indicating metastasis.

Mestrinho et al. (18) reported that the data of 159 patients with OSCC showed that PCNA was expressed in different degrees in all histological subtypes examined. Expression was related to the ages of patients and the stages of pathological lymph nodes. Most importantly, the high expression of $P C N A$ was a significant prognostic indicator for poor overall prognosis and disease-free survival of OSCC (21). In the acinar cell carcinoma subgroup, PCNA expression was found to be the only negative prognostic factor affecting the 5 -year tumor-free survival rate and overall survival (22). Simultaneously, the stability and overexpression of the $p 53$ gene might be related to $p 53$ gene mutation or genotoxic stress, and $p 53$ gene alterations are the most common gene abnormality in numerous cancers (23). Overexpression of $p 53$ plays an essential role in the development of OSCC (24).

Generally, mutation of the $p 53$ gene and overexpression of the mutant protein plays an important role in carcinogenesis and apoptosis in many human cancers. Simultaneously, $p 53$ mutations have been increasingly found in some poorly differentiated head and neck cancers, including an oral cavity in breast cancer $(20,21)$. Moreover, p53 changes are related to the invasive phenotype and recurrence of laryngeal and pharyngeal carcinomas. Overexpression of $p 53$ has been shown to be associated with a high risk of lymph node metastasis and is a marker of poor prognosis in oral cancer (22). The functional change of PCNA activity is a joint genetic event (23). $p 53$ is an effective marker of cell proliferation and could be used as an indicator to predict head and neck cancer. The overexpression of PCNA is also related to chemotherapy or the response to chemotherapy (24).

Since OSCC remains one of the most challenging cancers to control, with only slight improvement in survival over the past 50 years, prevention, treatment, and prognosis are crucial for OSCC. To improve the prognosis, survival biomarkers are needed. As our analysis demonstrated, $P C N A$ and $p 53$ might be suitable for prognostic and survival evaluation of OSCC. It was reported that expression of PCNA and P53 had association with some other kinds of carcinoma like skin cancer, colorectal cancer and lung cancer. There is need to analyze the other relationships in the future (20-23). It was also reported that PCNA and $\mathrm{Ki}-67$ were related to the abnormal proliferation of oral mucosa, and their proliferation index was parallel to the degree of proliferation, and they were linearly correlated $(25,26)$. So, we can conduct a further analysis between Ki67 and proliferation of OSCC in the next step.

However, there were some limitations in this study that should be noted. Firstly, more indicators evaluating other 
aspects between biomarkers and OSCC could be included, which should be conducted in the future. Secondly, comparisons between different subgroups, like age or area, could also be analyzed in future research.

\section{Acknowledgments}

Funding: None.

\section{Footnote}

Reporting Checklist: The authors have completed the PRISMA reporting checklist. Available at https://dx.doi. org/10.21037/atm-21-6133

Conflicts of Interest: All authors have completed the ICMJE uniform disclosure form (available at https://dx.doi. org/10.21037/atm-21-6133). The authors have no conflicts of interest to declare.

Ethical Statement: The authors are accountable for all aspects of the work in ensuring that questions related to the accuracy or integrity of any part of the work are appropriately investigated and resolved.

Open Access Statement: This is an Open Access article distributed in accordance with the Creative Commons Attribution-NonCommercial-NoDerivs 4.0 International License (CC BY-NC-ND 4.0), which permits the noncommercial replication and distribution of the article with the strict proviso that no changes or edits are made and the original work is properly cited (including links to both the formal publication through the relevant DOI and the license). See: https://creativecommons.org/licenses/by-nc-nd/4.0/.

\section{References}

1. Wu HT, Chen WT, Li GW, et al. Analysis of the Differentially Expressed Genes Induced by Cisplatin Resistance in Oral Squamous Cell Carcinomas and Their Interaction. Front Genet 2020;10:1328.

2. Kim K, Lee DJ. The updated AJCC/TNM staging system (8th edition) for oral tongue cancer. Transl Cancer Res 2019;8:S164-6.

3. Keshav R, Narayanappa U. Expression of Proliferating Cell Nuclear Antigen (PCNA) in Oral Submucous Fibrosis: An Immunohistochemical Study. J Clin Diagn Res 2015;9:ZC20-3.
4. Tang Q, Xie M, Yu S, et al. Periodic Oxaliplatin Administration in Synergy with PER2-Mediated PCNA Transcription Repression Promotes Chronochemotherapeutic Efficacy of OSCC. Adv Sci (Weinh) 2019;6:1900667.

5. Zhong LJ, Yuan F, Zhang W. Study on the expression of p53, p16 protein and PCNA in oral precancerous lesion and oral squamous cell carcinoma. Journal of Xinjiang Medical University 2002;9:53-9.

6. Wang X. Expressions of P27 protein and PCNA in oral squamous cell carcinomas. Journal of Practical Stomatology 2009;25:256-60.

7. Kadashetti V, Patil N, Datkhile K, et al. Analysis of expression of p53, p63 and proliferating cell nuclear antigen proteins in odontogenic keratocyst: An immunohistochemical study. J Oral Maxillofac Pathol 2020;24:273-8.

8. Fernanda CG, Sampaio-Góes DTOR. Expression of PCNA, p53, BAX, and BCL-X in oral poorly differentiated and basaloid squamous cell carcinoma: relationships with prognosis. Head Neck 2005;27:982-9.

9. Kato K, Kawashiri S, Yoshizawa K, et al. Expression form of $\mathrm{p} 53$ and PCNA at the invasive front in oral squamous cell carcinoma: correlation with clinicopathological features and prognosis. J Oral Pathol Med 2011;40:693-8.

10. Keum KC, Chung EJ, Koom WS, et al. Predictive value of $\mathrm{p} 53$ and PCNA expression for occult neck metastases in patients with clinically node-negative oral tongue cancer. Otolaryngol Head Neck Surg 2006;135:858-64.

11. Lee JJ, Kuo MY, Cheng SJ, et al. Higher expressions of p53 and proliferating cell nuclear antigen (PCNA) in atrophic oral lichen planus and patients with areca quid chewing. Oral Surg Oral Med Oral Pathol Oral Radiol Endod 2005;99:471-8.

12. Mallick S, Agarwal J, Kannan S, et al. PCNA and antiapoptotic Mcl-1 proteins predict disease-free survival in oral cancer patients treated with definitive radiotherapy. Oral Oncol 2010;46:688-93.

13. Monteiro LS, Diniz-Freitas M, Garcia-Caballero T, et al. Combined cytoplasmic and membranous EGFR and p53 overexpression is a poor prognostic marker in early stage oral squamous cell carcinoma. J Oral Pathol Med 2012;41:559-67.

14. Myoung H, Kim MJ, Lee JH, et al. Correlation of proliferative markers (Ki-67 and PCNA) with survival and lymph node metastasis in oral squamous cell carcinoma: a clinical and histopathological analysis of 113 patients. Int J 
Oral Maxillofac Surg 2006;35:1005-10.

15. Stenner M, Demgensky A, Molls C, et al. Prognostic value of proliferating cell nuclear antigen in parotid gland cancer. Eur Arch Otorhinolaryngol 2012;269:1225-32.

16. Watanabe S, Watanabe R, Oton-Leite AF, et al. Analysis of cell proliferation and pattern of invasion in oral squamous cell carcinoma. J Oral Sci 2010;52:417-24.

17. Sajeevan TP, Saraswathi TR, Ranganathan K, et al. Immunohistochemical study of p53 and proliferating cell nuclear antigen expression in odontogenic keratocyst and periapical cyst. J Pharm Bioallied Sci 2014;6:S52-7.

18. Mestrinho LA, Faísca P, Peleteiro MC, et al. PCNA and grade in 13 canine oral squamous cell carcinomas: association with prognosis. Vet Comp Oncol 2017;15:18-24.

19. Li L, Fukumoto M, Liu D. Prognostic significance of p53 immunoexpression in the survival of oral squamous cell carcinoma patients treated with surgery and neoadjuvant chemotherapy. Oncol Lett 2013;6:1611-5.

20. Lindemann A, Takahashi H, Patel AA, et al. Targeting the DNA Damage Response in OSCC with TP53 Mutations. J Dent Res 2018;97:635-44.

21. Carlos de Vicente J, Junquera Gutiérrez LM, Zapatero $\mathrm{AH}$, et al. Prognostic significance of $\mathrm{p} 53$ expression in oral squamous cell carcinoma without neck node metastases.

Cite this article as: Liu R, Sun K, Wang Y, Jiang Y, Kang J, $\mathrm{Ma} \mathrm{H}$. The effects of proliferating cell nuclear antigen and p53 in patients with oral squamous cell carcinoma: a systematic review and meta-analysis. Ann Transl Med 2021;9(23):1739. doi: $10.21037 / \mathrm{atm}-21-6133$
Head Neck 2004;26:22-30.

22. Ali SMA, Mirza Y. Overexpression of EGFR, COX2 and p53 in oral squamous cell carcinoma patients of Pakistan and correlation with prognosis. Ann Oncol 2019;30:VII21-VII22.

23. Kamat MS, Rai BD, Puranik RS, et al. Immunoexpression of p53 in histologically negative surgical margins adjacent to oral squamous cell carcinoma: A preliminary study. J Oral Maxillofac Pathol 2020;24:184.

24. Lu EM, Ratnayake J, Rich AM. Assessment of proliferating cell nuclear antigen (PCNA) expression at the invading front of oral squamous cell carcinoma. BMC Oral Health 2019;19:233.

25. Grzanka A, Sujkowska R, Janiak A, et al. Immunogold labelling of PCNA and Ki-67 antigen at the ultrastructural level in laryngeal squamous cell carcinoma and its correlation with lymph node metastasis and histological grade. Acta Histochem 2000;102:139-49.

26. Stenner M, Demgensky A, Molls C, et al. Prognostic value of PCNA expression in salivary gland cancer in consideration of different histological subtypes. Cancer Research 2011;71:Abstr 2956.

(English Language Editor: A. Kassem) 